\title{
Noncoding RNA-Mediated Chromatin Silencing (RmCS) in Plants
}

\section{Yuehui He*}

Department of Biological Sciences, National University of Singapore, Singapore

In a eukaryotic nucleus, DNA strands wrap around histones to form nucleosomes, the basic units of chromatin. Each nucleosome is composed of a histone octomer (two molecules of the highly conserved H2A, H2B, H3 and H4) wrapped by about 147-bp DNA. Both DNA and histones can be covalently modified. The cytosines of DNA can be methylated, whereas histone residues are often reversibly modified by acetylation, methylation, phosphorylation and/or ubiquitination. In addition, canonical nucleosomal histones can be replaced by histone variants, and nucleosomes are often remodeled (e.g. reposition) by ATPdependent chromatin-remodeling complexes. These modifications can regulate chromatin structure and modulate DNA accessibility to control gene expression and other DNA-templated activities.

A histone protein is composed of a structural core and an unstructured $\mathrm{N}$-terminal tail that is subject to various modifications. Depending on the position of a modified residue and the modification nature, histone modifications may lead to promotion or prevention of gene expression. For instance, histone acetylation, histone $\mathrm{H} 3$ lysine-4 (H3K4) methylation, H3K36 methylation and $\mathrm{H} 2 \mathrm{~B}$ monoubiquitination are associated with active gene expression, whereas histone deacetylation, H3K9 dimethylation (H3K9me2), $\mathrm{H} 3 \mathrm{~K} 27$ trimethylation (H3K27me3) and $\mathrm{H} 2 \mathrm{~A}$ monoubiquitination are often linked to gene silencing. Histone-modifying enzymes function either as a 'writer' or an 'eraser' to introduce or remove a modification, respectively [1]. A chromatin mark is typically recognized and bound specifically by a 'reader' and further interpreted by an 'effector' protein or protein complex [1], leading to a change in the chromatin state of a particular locus, which may cause gene activation or repression.

Cytosine DNA methylation occurs in a wide variety of eukaryotes. In animals such as mammals, cytosine methylation primarily is in the CG context, whereas in plants it also occurs in $\mathrm{CHG}$ and $\mathrm{CHH}(\mathrm{H}=\mathrm{A}$, $\mathrm{T}$ or $\mathrm{C}$ ) in addition to CG. DNA methylation is critical to silence transposons, repetitive elements and other invasive DNA elements, and thus maintain genome stability and integrity. In addition, cytosine methylation in a promoter region of a gene often causes gene silencing. In plants, the CG and CHG methylation during cell division are maintained by the highly-conserved DNA Methyltransferase 1 (MET1) and the plant-specific chromomethylase 3 (CMT3), respectively, and the maintenance of $\mathrm{CHH}$ methylation requires small RNAs (for target selection) and Domains-Rearranged Methyltransferase 2 (DRM2) [2].

The non-coding small RNAs (siRNAs) trigger de novo $\mathrm{CHH}$ methylation through two pathways in plants, including the wellcharacterized siRNA-directed DNA methylation (RdDM) pathway and the NERD pathway (NERD for 'Needed for RDR2-independent DNA methylation') [3,4]. In RdDM, RDR2 (for RNA-Dependent RNA Polymerase 2)-dependent 24-nt siRNAs are loaded to an ARGONAUTE 4 (AGO4)-containing effector complex, which associates with DRM2 and is guided to target loci by the siRNAs, resulting in cytosine methylation. In the NERD pathway, RDR1 or RDR6-dependent siRNAs such as 21-nt siRNAs, are loaded to an AGO2 effector complex; NERD, a GW repeat- and PHD finger-containing protein, directly interacts with AGO2 and target chromatin as well, and conceivably works together with the siRNAs to guide the effector complex to target loci, leading to cytosine methylation [3]. In addition to $\mathrm{CHH}$ methylation, the NERD pathway is also involved in CG and CHG methylation in a subset of non-conserved loci, as revealed in a study using the model plant Arabidopsis thaliana [3].

At the RdDM target loci, there are not only silencing cytosine methylations, but also repressive chromatin modifications such as histone deacetylation and $\mathrm{H} 3 \mathrm{~K} 9 \mathrm{me} 2$, and these modifications together establish a repressive chromatin environment at a target locus for its silencing [4], in which cytosine methylations promote or facilitate repressive histone modifications, and vice versa. In addition, at the NERD target loci, the NERD protein not only mediates cytosine methylation, but also recognizes and binds to un-methylated $\mathrm{H} 3 \mathrm{~K} 4$, and maintains lower levels of H3K4 methylation (note that elevated levels of this mark are associated with gene expression), to enforce silencing of its target loci [3]. Repressive histone/chromatin modifications are known to be required for DNA methylation. A few chromatin modifiers have been shown to be required for de novo and/ or maintenance of cytosine methylation, among which are the ATPdependent chromatin remodeler DDM1, the H3K9 methyltransferase KYP, Histone Deacetylase 6, and an H2B deubiquitinase called SUP32 [4]; in addition, several H3K4 demethylases known as LDL1, LDL2 and JMJ14/PKDM7B are also partly required for cytosine methylation [5-7]. Obviously, siRNA-triggered silencing of the RdDM and NERD target loci involves not only DNA methylation, but also repressive histone modifications. In addition to siRNAs, long non-coding RNAs (lncRNAs; with >200 nts) can also trigger chromatin silencing at their target loci. These chromatin-silencing mechanisms are referred hereafter as to non-coding RNA-mediated Chromatin Silencing (RmCS).

In the lncRNA-triggered RmCS, the RNA trigger causes repressive histone modifications such as $\mathrm{H} 3 \mathrm{~K} 27 \mathrm{me} 3$ (catalyzed by Polycomb repressive complex 2; PRC2) for silencing, which may not involve cytosine methylation. For instance, in Arabidopsis the COLDAIR lncRNA-triggered chromatin silencing of a central floral repressor known as FLC, involves H3K27me3, but not cytosine methylation [8]. In response to a prolonged cold exposure, COLDAIR expression is induced from the first intron of $F L C$, and subsequently, this lncRNA recruits a PRC2-like complex to FLC chromatin to deposit H3K27me3, leading to FLC silencing. A similar silencing mechanism has been observed in mammals: the mammalian HOTAIR lncRNAs spark X chromosome silencing via the recruitment of a PRC2 complex to the target chromosome, leading to its silencing or inactivation [9].

Genome-wide transcriptional analyses have identified thousands of $\operatorname{lncRNAs}$ in human and the Arabidopsis plant as well [10,11]. Some of these lncRNAs are likely to be involved in chromatin silencing.

${ }^{*}$ Corresponding author: Dr. Yuehui He, Department of Biological Sciences, National University of Singapore, Singapore 117543, Temasek Life Sciences Laboratory, Singapore 117604, Tel: +65 6872-7978; E-mail: yuehui@tll.org.sg

Received December 10, 2012; Accepted December 10, 2012; Published December 12, 2012

Citation: He Y (2013) Noncoding RNA-Mediated Chromatin Silencing (RmCS) in Plants. Mol Biol 2:e106. doi:10.4172/2168-9547.1000e106

Copyright: (c) $2013 \mathrm{He} \mathrm{Y}$. This is an open-access article distributed under the terms of the Creative Commons Attribution License, which permits unrestricted use, distribution, and reproduction in any medium, provided the original author and source are credited. 
RmCS triggered by long (lncRNAs) or short (siRNAs) non-coding RNAs, is emerging as an important chromatin mechanism underlying eukaryotic gene regulation.

\section{References}

1. Ruthenburg AJ, Allis CD, Wysocka J (2007) Methylation of lysine 4 on histone $\mathrm{H} 3$ : intricacy of writing and reading a single epigenetic mark. Mol Cell 25: 15-30.

2. Law JA, Jacobsen SE (2010) Establishing, maintaining and modifying DNA methylation patterns in plants and animals. Nat Rev Genet 11: 204-220.

3. Pontier D, Picart C, Roudier F, Garcia D, Lahmy S, et al. (2012) NERD, a PlantSpecific GW Protein, Defines an Additional RNAi-Dependent Chromatin-Based Pathway in Arabidopsis. Mol Cell 48: 121-132.

4. Chinnusamy V, Zhu JK (2009) RNA-directed DNA methylation and demethylation in plants. Sci China C Life Sci 52: 331-343.

5. Jiang D, Yang W, He Y, Amasino RM (2007) Arabidopsis relatives of the human lysine-specific Demethylase1 repress the expression of FWA and FLOWERING LOCUS C and thus promote the floral transition. Plant Cell 19: 2975-2987.
6. Deleris A, Greenberg MV, Ausin I, Law RW, Moissiard G, et al. (2010) Involvement of a Jumonji-C domain-containing histone demethylase in DRM2mediated maintenance of DNA methylation. EMBO Rep 11: 950-955.

7. Searle IR, Pontes O, Melnyk CW, Smith LM, Baulcombe DC (2010) JMJ14, a $\mathrm{JmjC}$ domain protein, is required for RNA silencing and cell-to-cell movement of an RNA silencing signal in Arabidopsis. Genes Dev 24: 986-991.

8. Heo JB, Sung S (2011) Vernalization-mediated epigenetic silencing by a long intronic noncoding RNA. Science 331: 76-79.

9. Jeon Y, Sarma K, Lee JT (2012) New and Xisting regulatory mechanisms of X chromosome inactivation. Curr Opin Genet Dev 22: 62-71.

10. Liu J, Jung C, Xu J, Wang H, Deng S, et al. (2012) Genome-Wide Analysis Uncovers Regulation of Long Intergenic Noncoding RNAs in Arabidopsis. Plant Cell.

11. Volders PJ, Helsens K, Wang X, Menten B, Martens L, et al. (2012) LNCipedia: a database for annotated human IncRNA transcript sequences and structures. Nucleic Acids Res. 